\title{
EVENT-RELATED POTENTIALS ELICITED BY A VISUAL CONTINUOUS PERFORMANCE TASK IN CHILDREN OF ALCOHOLICS
}

Authors: Socorro Rodríguez Holguín, Montserat Corral and Fernando Cadaveira

This is the peer reviewed version of the following article: Rodríguez Holguín, S.; Corral, M.; Cadaveira, F. (1999). Event-related potentials elicited by a visual continuous performance task in children of alcoholics. Alcohol, 19, 23-30. doi: 10.1016/S0741-8329(99)00013-0

This article may be used for non-commercial purposes in accordance with Elsevier Masson terms and conditions for use of self-archived versions. 


\title{
EVENT-RELATED POTENTIALS ELICITED BY A VISUAL CONTINUOUS PERFORMANCE TASK IN CHILDREN OF ALCOHOLICS
}

\author{
Authors: Socorro Rodríguez Holguín, Montserat Corral and Fernando Cadaveira \\ Department of Clinical Psychology and Psychobiology, University of Santiago de Compostela, \\ Galicia, Spain \\ Requests for reprints should be addressed to Departamento de Psicoloxía Clínica e Psicobioloxía, \\ Facultade de Psicoloxía, Campus Universitario Sur, E-15706, Santiago de Compostela, Galicia, \\ Spain. Tel: + 34-981-563100 (ext. 13798); Fax: + 34-981-521581; E-mail: holguin@usc.es
}




\begin{abstract}
Event-related potentials (ERPs) were recorded from a group of young children of alcoholics (HR; $n-17,7$ females) with a high-density family-history of alcoholism and from a control group (CN; $n-19,10$ females), ages $7-15$ years old, during a visual continuous performance task. The P3 peak amplitude and the mean amplitude at five latency windows (300-800 ms) were measured at frontal (F3-Fz-F4), central (C3-Cz-C4) and parietal (P3-Pz-P4) electrodes. Data were analyzed using a mixed-model risk-group by stimulus-type (matching vs. nonmatching) by Electrode ANCOVA, with age as a covariate, for each of the scalp regions. The risk-group by stimulustype interactions were significant at the parietal region for the $\mathrm{P} 3$ peak amplitude and for the 300 $400 \mathrm{~ms}$ mean amplitude, although there were no risk-group main differences. The HR group manifested smaller differences between the amplitude of the matching and nonmatching condition than the $\mathrm{CN}$ group. These results suggest a deficient electrophysiological differentiation between relevant and irrelevant information and are discussed in relation to previous reports and to the characteristics of the sample
\end{abstract}


THE relevance of the study of the neurocognitive factors related to the familial transmission of alcoholism is clear from the increasing number of reports about this subject in recent years. Some laboratories have focused on the identification of psychophysiological and neuropsychological variables that would identify subpopulations at increased risk for alcoholism and have mainly assessed children of alcoholic fathers. One area of investigation has evaluated the effects of acute alcohol administration on these variables. Alcohol has differential effects on children of alcoholics over autonomic variables in response to stressful or novel stimuli, with these subjects appearing more sensitive to the stress-dampening effects of alcohol $(12,17)$. With regard to CNS measures, a pioneering study (14) found that adult children of alcoholics were more sensitive than controls to the effect of acute alcohol administration on the P3 component of the ERPs during attention tasks, and differences in other event-related potential (ERP) components (e.g., N100) have also been reported (5). Differential effects of alcohol on neuropyschological tests performance have also been studied, although the results are less consistent $(18,25)$.

Other investigations have studied the differences between healthy children of alcoholics and controls without alcohol administration. They have the advantage of allowing the assessment of young, alcohol-naive subjects, to search for variables that characterize subjects at risk for alcoholism without interaction with consumption. Biochemical (serotonin metabolites, MAO-B), psychophysiological [heart rate, electrodermal response, electroencephalographic (EEG), ERPs], neuropsychological (visuospatial abilities, attention, memory, planning), behavioral (hyperactivity, conduct problems) and personality (sensation seeking, reward dependence) variables have been studied and reviewed in literature $(15,25,28)$. One important branch of this area of research is that focused on functioning in children of alcoholics. Begleiter and colleagues reported that young sons of alcoholic fathers manifested a diminished voltage in the P3 component of the visual ERPs, in the same way as alcoholic subjects (6). Subsequently, several other laboratories have assessed the P3 component of ERPs elicited by visual and auditory tasks with different levels of difficulty, with samples composed of young and adult children of alcoholics.

In 1994, a meta-analysis study of 22 reports that compared the P3 of non-alcoholic subjects with and without a family history of alcoholism was published (20). It lead to the conclusion that the P3 voltage is smaller in subjects at family risk for alcoholism and suggested that several moderator factors contribute to explaining discrepant findings among laboratories. The age of the samples, the sensory modality assessed, and the difficulty of the tasks used to elicit P3 appeared to be the principal moderating factors. Polich and co-workers stated that differences between the risk and control groups are more robust when difficult visual tasks are used to assess young subjects (20). Nonetheless, they also recommended to pay attention in future empirical studies to other factors such as the source of recruitment, the presence of additional Axis I or Axis II disorders in the families, or the neuropsychological performance in attention and memory processes. The relevance of these factors has also been pointed out by Begleiter and Porjesz (5). In addition, two recent follow-up studies $(9,16)$ point that ERP abnormalities in childhood appear useful as good predictors of adolescent alcohol and drugs abuse.

In our laboratory, young children of alcoholics were classified according to the presence or absence of a multigenerational family history of alcoholism; those with other psychopathological diseases in first- or second-degree relatives were excluded. They were assessed with a set of paradigms with varying levels of difficulty to elicit several ERP components, as well as with a

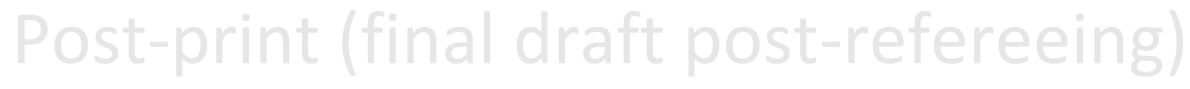


battery of neuropsychological tests. When an easy discrimination visual task was employed, the P3 elicited by a target had lower amplitude and longer latency only in the female subgroup with a multigenerational family history of alcoholism, but there were no differences with the control group for the males (23). The P3 elicited by the infrequent nontarget stimuli did not differ in amplitude between children of alcoholics and controls, although latency differences were observed (22).

In the present report, ERPs were assessed using a more complex visual task. The paradigm used replicates the continuous performance task as implemented by Noble and colleagues $(8,26,27)$. This paradigm was selected because it involves a more complex processing of stimuli than discrimination tasks. As stated above, this has been considered a moderating factor of differences in ERPs between children of alcoholics and controls (20). Moreover, this paradigm has been used by Whipple and colleagues to study the relationship between electrophysiological measures and neuropsychological achievement in visuoperceptual and memory tests (26).

\section{Method}

\section{Subjects}

The subjects were 36 males and females ranging from 7 to 15 years of age. The high-risk (HR) group $\left(\mathrm{n}=17,7\right.$ females, mean $=11.8^{+}-2.3 \mathrm{yr}$ ) consisted of children of alcoholic fathers with a high density family history of alcoholism. The subjects in the HR group were selected from community treatment centres, where their fathers had been diagnosed and treated. All the alcoholic fathers met DSM-III-R (2) criteria for alcohol dependence. (A diagnosis made by the staff of the centres was corroborated during the selection interview.) Those with a history of psychopathological problems other than secondary to alcoholism (according to the clinical history from the centres and the information collected during the selection interview) were excluded. The family history of alcoholism was ascertained from fathers and mothers using the family history interview method. Only children of alcoholic fathers who had at least two other first- or seconddegree alcoholic relatives were included. The control $(\mathrm{CN})$ group $(\mathrm{n}=19,10$ females, mean $=$ $12.0+-1.7 \mathrm{yr}$ ) consisted of children of non alcoholic fathers without a family history of alcoholism. To guarantee homogeneity with regard to sociodemographic variables, control subjects were recruited from voluntary families from schools in the region within the same age range and socioeconomic status as those in the HR group. Control families who reported any problems with alcohol in first- or second- degree relatives were excluded.

Other exclusionary criteria were similar for the two groups and included consumption of alcohol or other drugs, a history of psychopathological disorders, prenatal exposure to alcohol, developmental or school retardation, a positive neurological history, major medical problems, current medication, noncorrected sensory deficits, a family history of major mental diseases, and problems of alcoholism in the mother. Information about inclusion and exclusion criteria was obtained through detailed semi-structured interviews with both the children and their fathers and mothers. The interviews were a translated and adapted version of the Semi-Structured Assessment for the Genetics of Alcoholism versions for adults, children, adolescents, and parents, as well as the Family History Assessment Module, designed by the Collaborative Study on the Genetics of Alcoholism (11). Questions about individual and familial psychopathological problems were based on DSMIII- R criteria and at least one other diagnostic classification system. Information

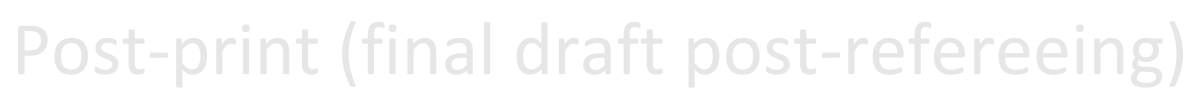


was also obtained during the interviews about demographic data, family relations, school achievement and social activities.

The final sample was well matched for age, handedness, socio-economic status and educationall subjects were enrolled in compulsory schooling and followed the grade according to agebetween the groups (see Table 1). Subjects from the two groups were randomly distributed across environmental variables such as ERPs assessment time (time of day, month), or recency of food ingestion (19).

Families who met the requirements for the study were asked to participate; those who agreed signed a consent form and received an appointment for the assessment. When the children arrived at the laboratory (early in the morning or in the afternoon), the members of staff showed them the laboratory and explained the contents and procedure of the assessment.

Once electrodes had been put in place, subjects sat in a comfortable armchair, in an electrically isolated, sound- and light-attenuated laboratory. They received general instructions to avoid moving during the test and to pay attention to the instructions about the task.

The visual continuous performance task is a replica of that designed by Noble et al. (26). Subjects were instructed to watch to a video monitor placed $1 \mathrm{~m}$ in front of them, where visual stimuli were presented. The stimuli, presented one at atime, at a constant ISI (onset-onset) of $2.1 \mathrm{~s}$ with a duration of $100 \mathrm{~ms}$ subtended a visual area of $4.8^{\circ} \mathrm{X} 4.8^{\circ}$. The stimuli varied in three dimensions: shape, color, and the identity of a numeral in the center of each shape. Circles, squares, and triangles, which were either orange, blue, green, or violet and contained a numeral between 0 and 9 , were used. Subjects had to press a button with the preferred hand when two consecutive stimuli matched in all the three stimulus dimensions. The series consisted of 200 stimuli, with matches occurring pseudo-randomly with the only restriction of no two matches appearing consecutively, and a probability of 0.11 ( $n=22$ matches).

\section{ERP Recording}

EEG activity was recorded at nine scalp sites: Fz, F3, F4, Cz, C3, C4, Pz, P3, and P4 (standard electrode position nomenclature)(1), using tin electrodes inserted in an electrocap (Electro-Cap International, Inc.), referred to linked earlobes, and with a forehead ground. Additional electrodes were used to monitor eye movements (supraorbital and the outer canthus of the left eye, referred to an infraorbital electrode). EEG activity was filtered $(0.1-30 \mathrm{~Hz})$ and amplified $10 \mathrm{~K}$ (Grass Neurodata Acquisition System, model 12, connected to a Neuro Scan, Inc. system for the analogue-to-digital conversion and storage). Impedance values were kept at $5 \mathrm{KV}$ or below.

EEG was continuously sampled at a rate of $256 \mathrm{~Hz}$. The signal was processed off-line. First, EEG was corrected for ocular artefacts, using the algorithm developed by Semlitsch and colleagues (24); then EEG was epoched from $100 \mathrm{~ms}$ prestimulusto $900 \mathrm{~ms}$ poststimulus, linear trends were eliminated, and the signal was adjusted to $0 \mathrm{~m} \mathrm{~V}$ prestimulus baseline. Trials exceeding $685 \mathrm{~m} \mathrm{~V}$ at any scalp electrode were identified by visual inspection and rejected. The epochs corresponding to incorrect responses (omissions or false alarms) were also rejected. Finally, trials were averaged according to type of stimuli (matches and nonmatches), and digital filtering was performed offline using a $0.1-16 \mathrm{~Hz}$ band-pass filter. Only those subjects $(n=36)$ with at least 15 epochs for the averaged waveform elicited by matches were included in the study; 14 subjects from the initial sample (seven at each risk group) were dropped because of less than 15 epochs remained for 
averaging. The number of target epochs in the HR (mean $=18.1+-2.3$, range $=15-22)$ and the $\mathrm{CN}$ (mean $=18.0+-1.8$, range 15-22) groups did not differ $(t=0.10, p>.0 .924)$.

\section{Data Analysis}

ERPs were automatically measured for both the matchingand the nonmatching recordings, as the mean amplitudes $(\mu \mathrm{V})$ in five 100 ms poststimulus intervals: $300-400,400-500,500-600,600$ 700 , and $700-800 \mathrm{~ms}$. P3 peak amplitude $(\mu \mathrm{V})$ was also measured, and identified, using a computer algorithm, as the maximum positive peak at each electrode between 400 and $600 \mathrm{~ms}$; peaks were then verified and adjusted by visual inspection, and those which were doubtful were revised by a second experienced member of the laboratory,blind to the risk status of the subject and the initial peak. Amplitude values were automatically exported to an ASCII file for subsequent analyses.

The ERP measurements were organised into three electrode groupings: frontal (F3, F4, Fz), central $(\mathrm{C} 3, \mathrm{C} 4, \mathrm{Cz})$, and parietal $(\mathrm{P} 3, \mathrm{P} 4, \mathrm{Pz})$. Preliminary risk group by gender and risk group by age analyses were made for determining the inclusion of gender and age variables in the design. As there were no significant interactions in these analyses and no significant differences between males and females, both genders were considered jointly, and age was included as a covariate. Therefore, a 2 X 2 X 3, risk group (CN vs. HR) by stimulus type (matching vs. nonmatching) by electrode mixed-model ANCOVA, with the risk group as a between-subjects factor, the stimulustype and the electrode as within-subject factors, and age as a covariate were used to assess group differences in the ERPs mean amplitude at each time interval and in the P300 peak amplitude in each of the electrode groupings. Degrees of freedom were corrected by the conservative Greenhouse- Geisser estimate when appropriate. Moreover, to avoid that risk group differences at individual electrodes may bemasked by regional analyses, those electrodes where the P3 component is more frequently assessed, $\mathrm{Pz}$ and $\mathrm{Cz}$ were separately analyzed, using a risk group by stimulus-type ANCOVA. Behavioural data (response time and percentage of correct responses) were assessed using an ANCOVA comparison between the risk groups with age as a covariate.

\section{Results}

\section{Behavioral Performance}

Table 2 summarizes the behavioral data for each group. No significant differences between the risk groups were observed for response time and percentage of correct responses $(p .0 .05)$.

\section{ERP Measurements}

Figure 1 illustrates the grand mean waveforms for the two risk groups in the two stimulus types at each of the electrodesrecorded. The descriptive estatistics of the data are summarized in Table 3.

The risk group between-subjects factor manifested no significant differences at any of the mean amplitude intervals or the $\mathrm{P} 3$ peak amplitude values.

The stimulus-type within-subjects factor was significant over the whole sample at the parietal region $(\mathrm{P} 3, \mathrm{Pz}, \mathrm{P} 4)$ for all the mean amplitude intervals except the $300-400$ and the $700-800 \mathrm{~ms}$ intervals: $400-500 \mathrm{~ms}: F(1,34)=7.74, p<0.009 ; 500-600 \mathrm{~ms}: F(1,34)=21.83, p<0.0005 ; 600$ 
700 ms: $F(1,34)=22.15, p<0.0005$. At the central region $(\mathrm{C} 3, \mathrm{Cz}, \mathrm{C} 4)$ this factor was significant for all except the 700-800 ms mean amplitude interval: $300-400 \mathrm{~ms}: F(1,34)=7.18, p<0.011$; 400-500 ms $F(1,34)=7.64, p<0.009$; 500-600 ms: $F(1,34)=15.62, p<0.0005 ; 600-700 \mathrm{~ms}: F$ $(1,34)=11.22, p<0.002$. Finally, at the frontal region $(\mathrm{F} 3, \mathrm{Fz}, \mathrm{F} 4)$ it was significant only for the earlier mean amplitude intervals: $300-400 \mathrm{~ms}: F(1,34)=13.17, p<0.001$; and 400-500 ms: $F(1$, $34)=8.29, p<0.007$. The P3 peak amplitude was significantly different for the matching and the non-matching stimuli at the parietal $[F(1,34)=32.69, p<0.0005]$, central $[F(1,34)=23.19$, $p<0.0005]$, and frontal $[F(1,34)=17.54, p<0.0005]$ regions. In all these variables, the amplitudes elicited by the matching stimuli were larger than those elicited by the nonmatching stimuli.

The risk group by stimulus-type interactions were significant only at the parietal region for the $300-400$ ms mean amplitude interval $[F(1,34)=4.75, p<0.036]$ and for the $\mathrm{P} 3$ peak amplitude at the same scalp region $[F(1,34)=5.59, p<0.024]$. To clarify the meaning of interactions, simple effects analyses were made. The comparison of the two stimulus types separately for each risk group indicated that, for the 300-400 ms mean amplitude interval, the ERPs elicited at the parietal region by the matching stimuli were larger than those elicited by the nonmatching stimuli in the $\mathrm{CN}$ group $[F(1,18)=4.70, p<0.044]$, but there were no stimulus-type differences in the HR group $[F(1,16)=0.82, p<0.380]$. With regard to the $\mathrm{P} 3$ parietal amplitude, the stimulustype comparisons were significant for both the $\mathrm{CN}[F(1,18)=30.74, p<0.0005]$ and the HR $[F(1,16)=6.20, p<0.024]$ groups, although the difference between the amplitude of the matching and the nonmatching condition was smaller in the HR than in the $\mathrm{CN}$ group $[F(1,33)=5.54, p$ $<0.025]$ (see Figure 2).

The electrode within-subject factor was significant $(p<0.05)$ at the three scalp regions and the five mean amplitude intervals except for the frontal electrode grouping at the 500- $600 \mathrm{~ms}$ and the $700-800 \mathrm{~ms}$ intervals, and for the parietal electrode grouping at the 700-800 ms interval. Frontal region: $300-400 \mathrm{~ms}: F(2,68)=3.47, p<0.037 ; 400-500 \mathrm{~ms}: F(2,68)=4.06, p<0.022 ; 600-700$ ms: $F(2,68)=4.45, p<0.015$. Central region: $300-400 \mathrm{~ms}: F(2,68)=10.96, p<0.0005 ; 400$ $500 \mathrm{~ms}: F(2,68)=11.14, p<0.0005 ; 500-600 \mathrm{~ms}: F(2,68)=9.22, p<0.0005 ; 600-700 \mathrm{~ms}: F(2$, $68)=10.38, p<0.0005 ; 700-800 \mathrm{~ms}: F(2,68)=18.18, p<0.0005$. Parietal region: $300-400 \mathrm{~ms}:$ $F(2,68)=6.70, p<0.002 ; 400-500 \mathrm{~ms}: F(2,68)=15.56, p<0.0005 ; 500-600 \mathrm{~ms}: F(2,68)=$ $24.35, p<0.0005$; 600-700 ms: $F(2,68)=15.82, p<0.0005$. The electrode factor was also significant for the P3 peak amplitude at the central $[F(2,68)=18.67, p<0.0005]$ and parietal $[F(2,68)=25.58, p<.0005]$ regions. The electrode differences reflected that the maximum amplitudes were recorded at the midline electrodes in the three regions $(\mathrm{Pz}, \mathrm{Cz}, \mathrm{Fz})$ compared with the lateral electrodes.

The risk group by electrode interactions were not significant for any of the dependent variables considered at this report.

Finally, the covariate (age), was significant at the following variables: Parietal: $300-400 \mathrm{~ms}$ ( $p<$ $0.010)$; 400-500 ms $(p<0.019) ; 700-800 \mathrm{~ms}(p<0.010)$. Central: 300-400 ms $(p<0.0005)$; 400$500 \mathrm{~ms}(p<0.003) ; 600-700 \mathrm{~ms}(p<0.048) ; 700-800 \mathrm{~ms}(p<0.0005)$. Frontal: 300-400 ms $(p<$ $0.0005) ; 400-500 \mathrm{~ms}(p<0.008) ; 600-700 \mathrm{~ms}(p<0.012) ; 700-800 \mathrm{~ms}(p<0.027)$. P3 amplitude at central $(p<0.038)$ and frontal $(p<0.021)$ regions. The amplitude increased with age for all these

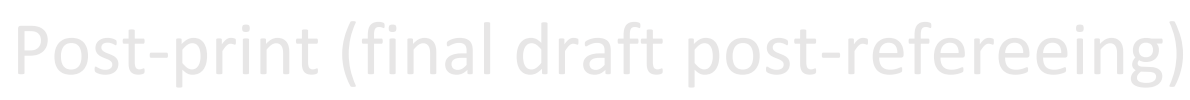


variables. A reanalysis without covariate in those cases, where it was not significant, did not modify the significance of the other factors.

\section{$C z$ and $P z$ Separate Analyses}

The individual analyses of $\mathrm{Cz}$ and $\mathrm{Pz}$ confirmed the effects observed in the regional analyses. There were no Risk Group main effects or Risk Group by Condiction interactions for any of the amplitude variables at the $\mathrm{Cz}$ electrode. The Risk Group by Condition interaction was significant for 300-400 ms amplitude $[F(1,34)=5.55, p<0.024]$ and for the P3 peak amplitude $[F(1$, $34)=6.54, p<0.015]$ at $\mathrm{Pz}$, due to the smaller differences between matching and nonmatching amplitude in the HR group.

\section{Discussion}

In this study, the overall measures of ERP amplitudes were no different between children of alcoholic fathers with a multigenerational family history of alcoholism and controls, although significant differences involving the risk group factor appeared in the interaction with the stimulus type (matching vs. nonmatching condition) in the parietal region, affecting the $\mathrm{P} 3$ peak amplitude and the mean amplitude in the 300-400 ms interval. The main effects of the within-subjects factors, electrode and stimulus condition were those expected in this paradigm: maximum amplitudes at the midline electrodes in all the scalp regions and larger amplitudes elicited by the matching than the nonmatching stimuli.

These results affecting the risk groups should be discussed in relation to the previous general literature that has reported a diminished voltage in the $\mathrm{P} 3$ of children of alcoholics but specifically in relation to the results using the same paradigm with other samples (bearing in mind that the previous results used other paradigms with this sample). The first report by Whipple et al. (26) using the paradigm replicated here compared the mean amplitude for the ERPs to the matching stimuli at 300-400, 400-500 and 500-600 ms latency windows at Fz, Cz, and Pz electrodes. They found that subjects with a family history of alcoholism manifested smaller amplitudes at the 300 400 and 400-500 ms latency windows at the three electrodes. Subsequent reports from the same laboratory confirmed the presence of differences between high-risk young subjects and controls. In a latter study (27), researchers found reduced amplitudes and elongated latencies of the P3 elicited by the matching stimuli at Pz, although the overall comparison, including the two stimulus condition and five electrodes, had been no-significant for the P3 amplitude and the slow wave mean amplitude. With a new sample and a more detailed analysis (8), these authors averaged the ERPs according to the number of matching features and found no significant main effects for the group factor but a significant group by stimulus condition (match level) interaction for 500 to 800 ms.

Therefore, although no main group differences in amplitude were observed, the results presented here are not discrepant with those previously obtained with the same paradigm. As may be seen in Figure 2, the risk group by stimulus interaction was due to a lesser differentiation between the voltage of the matching and the nonmatching waveforms in the high-risk group. This is a similar pattern to that describe by Berman and colleagues (8) and would corroborate the hypothesis that subjects at risk for alcoholism are less able to differentiate, at an electrophysiological level, between relevant and irrelevant information. If the P3-like positive components of ERPs are 
related to the neural inhibition necessary to limit cortical excitation to task specific areas $(10,21)$, these results would indicate that subjects at risk for alcoholism are less efficient in the distribution of attentional and memory resources between the relevant and irrelevant stimuli.

Whipple et al. (26) related the diminished voltage in the ERPs of high-risk subjects with the performance in neuropsychological tests of visuoperceptual abilities and memory and found that these subjects obtained lower scores in visuoperceptual tests. The neuropsychological assessment carried out at our laboratory, with a more extensive sample than that included in this report (13), indicated that children of alcoholics had significantly lower scores in the block design subtest of the Weschler Intelligence Scale (WISC-R). This is an interesting fact as this was one of the tasks that Whipple and colleagues correlated with the electrophysiological data and is coherent with the data that support the fact that the anomalies in ERPs are most consistent in the visual modality.

Nonetheless, it should be pointed out that when only the subsamples used here were analyzed, the differences in the block design subtest scores did not reach statistical significance $(p<0.066)$. Although the size of the sample studied here is similar to that reported by Whipple and colleagues, this loss of significance in the block design subtest when the sample was reduced indicates the need for increasing the number of subjects studied in the assessment of ERPs in order to achieve more significant results.

Another important question refers to the selection criteria of the high-risk group. The children of alcoholics selected in the present study had at least three alcoholic relatives in the paternal family, and other psychopathological disorders were absent both in the alcoholic and control families. The absence of antisocial personality traits in alcoholic fathers, or conduct problems in children, is especially relevant. Bauer and colleagues found that a family history of alcoholism and antisocial personality disorder may have additive or interactive effects on the P3 amplitude, with both factors contributing to the diminished voltage of this component in the high-risk subjects $(3,4)$. Another relevant factor that has been indicated is family stress because subjects with DRD2 deficience (that has been associated with severe alcoholism) manifest a negative correlation between P3 amplitude and family stress (7). It has been proposed that this genetic-environmental interaction could explain the discrepant results in high-risk studies. This variable has not been systematically addressed in the present study. Perhaps the absence of main group effects on the voltage measurements is related to these psychopathological, genetical, and environmental factors, and they should be systematically assessed in a future extension of the present research.

In summary, this study confirms the presence of some electrophysiological differences between young children of alcoholics and controls during the execution of a visual continuous performance task. Even though the results do not reach the strength of those reporting overall decrements in P3 amplitudes (26-27), they agree with findings of group by match level interactions (8). The presence of risk group by stimulus type interactions for P3 peak amplitude and for the 300-400 ms mean interval amplitude would suggest a deficiency in the electrophysiological differentiation between relevant and irrelevant information that has already proposed in previous reports.It is possible that these differences between groups achieves a greater significance if other factors, such as conduct problems, antisocial traits or family stress are significantly present in the alcoholic families and should be assessed in future research.

\section{Acknowledgements}

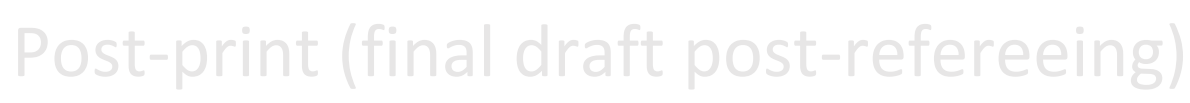


This research was supported by the Spanish Ministry of Education and Culture (DGICYT) grant PB95-0856. We thank the staff of UTA at the Hospital Xeral de Santiago de Compostela, Asociación de Ex- Alcohólicos de A Coruña and Asociación Viguesa de Alcohología for their help in recruiting of the sample.

\section{Reference}

1. American Electroencephalographic Society.: American Electroencephalographic Society guidelines for standard electrode position nomenclature. J. Clin. Neurophysiol. 8:200-202; 1991.

2. American Psychiatric Association: Diagnostic and Statistical Manual of Mental Disorders. Revised 3rd Ed. Washington, D.C.: American Psychiatric Association; 1987.

3. Bauer, L. O.; Hesselbrock, V. M.; O'Connor, S.; Roberts, L.: P300 differences between nonalcoholic young men at average and above-average risk for alcoholism: effects of distraction task and modality. Progr. Neuropsychopharmacol. Biol. Psychiatry 18:263-277; 1994.

4. Bauer, L. O.; O'Connor, S.; Hesselbrock, V. M.: Frontal P300 decrements in antisocial personality disorder. Alcohol. Clin. Exp. Res. 18:1300-1305; 1994.

5. Begleiter, H.; Porjesz, B.: Neurophysiological phenotypic factors in the development of alcoholism. In: Begleiter, H.; Kissin, B., eds. The genetics of alcoholism. New York: Plenum Press; 1995:269-293.

6. Begleiter, H.; Porjesz, B.; Bihari, B.; Kissin, B.: Event-related brain potentials in boys at risk for alcoholism. Science 225:1493-1496; 1984.

7. Berman, S. M.; Noble, E. P.: The D2 dopamine receptor (DRD2) gene and family stress, interactive effects on cognitive functions in children. Behav. Genet. 27:33-43; 1997.

8. Berman, S. M.; Martínez, R. A.; Noble, E. P.: Familial alcoholism and ERPs: differences in probability sensitivity? Alcohol Alcohol. 28:695-707; 1993.

9. Berman, S. M.; Whipple, S. C.; Fitch, R. J.; Noble, E. P.: P3 in young boys as predictor of adolescent substance use. Alcohol 10:69-76; 1993.

10. Birbaumer, N.; Elbert, T.; Canavan, A. G. M.; Rockstroh, B.: Slow potentials of the cerebral cortex and behavior. Physiol. Rev. 70:1-41; 1990.

11. Bucholz, K. K.; Cadoret, R.; Cloninger, C. R.; Dinwiddie, S. H.; Hesselbrock, V. M.; Nurnberger, J. I.; Reich, T.; Schmidt, I.; Schuckit, M. A.: A new, semi-structured psychiatric interview for use in genetic linkage studies: a report of the reliability of the SSAGA. J. Stud. Alcohol. 55:149-158; 1994.

12. Conrod, P. J.; Pihl, R. O.; Ditto, B.: Autonomic reactivity and alcohol-induced dampening in men at risk for alcoholism and men at risk for hypertension. Alcohol. Clin. Exp. Res. 19:482489; 1995.

13. Corral, M.; Rodríguez Holguín, S.; Cadaveira, F.: Neuropsychological characteristics in children of alcoholics: familial density. J. Stud. Alcohol. (in press). 
14. Elmasian, R.; Neville, H.; Woods, D.; Schuckit, M.; Bloom, F.: Event-related brain potentials are different in individuals at high and low risk for developing alcoholism. Proc. Natl. Acad. Scienc. USA. 79:7900-7903; 1982.

15. Galanter, M., ed.: Recent developments in alcoholism. Vol. 9. Children of alcoholics. New York: Plenum Press; 1991.

16. Hill, S. Y.; Steinhauer, S. R.; Lowers, L.; Locke, J.: Eight-year longitudinal follow-up of P300 and clinical outcome in children from high-risk for alcoholism families. Biol. Psychiatry. 37:823$827 ; 1995$.

17. Newlin, D. B.; Thomson, J. B.: Alcohol challenge with sons of alcoholics: a critical review and analysis. Psychol. Bull. 108:383- 402; 1990.

18. Peterson, J. B.; Finn, P. R.; Pihl, R. O.: Cognitive dysfuntcion and the inherited predisposition to alcoholism. J. Stud. Alcohol. 53:154- 160; 1992.

19. Polich, J.; Kok, A.: Cognitive and biological determinants of P300: an integrative review. Biol. Psychol. 41:103-146; 1995.

20. Polich, J.; Pollock, V. E.; Bloom, F. E.: Meta-analysis of P300 amplitude from males at risk for alcoholism. Psychol. Bull. 115:55- 73; 1994.

21. Rockstroh, B.; Muller, M.; Cohen, R.; Elbert, T.: Probing the functional brain state during P300-evocation. J Psychophysiol. 6:175-184; 1992.

22. Rodríguez Holguín, S.; Corral, M.; Cadaveira, F.: Event-related potentials elicited by infrequent non-target stimuli in young children of alcoholics. Family history and gender differences. Alcohol Alcohol. 33:281-290; 1998.

23. Rodríguez Holguín, S.; Corral, M.; Cadaveira, F.: Visual and auditory event-related potentials in young children of alcoholics from high- and low-density families. Alcohol. Clin. Exp. Res. 22:87-96; 1998 .

24. Semlitsch, H. V.; Anderer, P.; Schuster, P.; Presslich, O.: A solution for reliable and valid reduction of ocular artifacts applied to the P300 ERP. Psychophysiol. 23:695-703; 1986.

25. Sher, K. J.: Children of alcoholics. A critical appraisal of theory and research. Chicago: Chicago University Press; 1991.

26. Whipple, S. C.; Parker, E. S.; Noble, E. P.: An atypical neurocognitive profile in alcoholic fathers and their sons. J. Stud. Alcohol. 49:240-244; 1988.

27. Whipple, S. C.; Berman, S. M.; Noble, E. P.: Event-related potentials in alcoholic fathers and their sons. Alcohol. 8:321-327; 1991.

28. Windle, M.; Searles, J. S., eds.: Children of alcoholics. Clinical perspectives. New York: The Guildford Press; 1990 


\section{TABLE 1}

DHMOGRAPHC CHARACIHRITITS OF CONIROL AND HIGH-RISK GROLPS

\begin{tabular}{|c|c|c|c|}
\hline & $\begin{array}{l}\text { Controbi } \\
(\mathrm{m}=1 \%)\end{array}$ & $\begin{array}{c}\text { Hgh Rek } \\
\text { (n }=17)\end{array}$ & $\boldsymbol{P}$ \\
\hline Ciender (fim) & 109 & 710 & $1492^{*}$ \\
\hline Age (monge) & $7-15$ & $7-15$ & \\
\hline Mean $(S D)$ & $120(1.7)$ & $118(23)$ & 0792 \\
\hline fermales & $123(1.5)$ & $119(27)$ & 0956 \\
\hline males & $11.7(1.5)$ & $11.8(2.5)$ & 0977 \\
\hline I-Thest f w in (p) & 0773 & 0962 & \\
\hline Mean Alooholix relatives & 0 & 33 & - \\
\hline Fducation (years) & $63(2.9$ & $64(1.6)$ & 0922 \\
\hline Handodness (RT/A) & 1720 & 16110 & $0542^{*}$ \\
\hline
\end{tabular}

Ehi gquare comparimon.

TABLE 2

BHHAVDRAL DATA FOR CONTIOL AND HIH-RISE GROUP

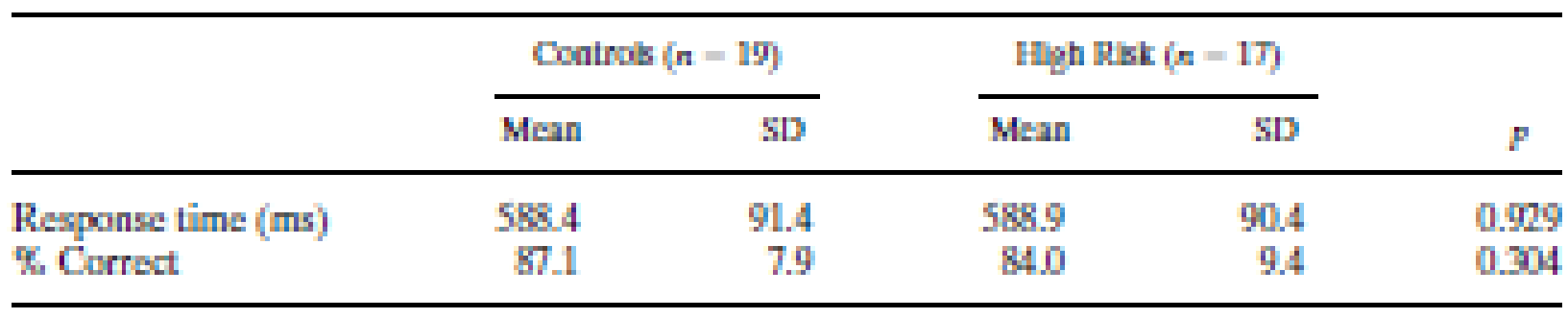



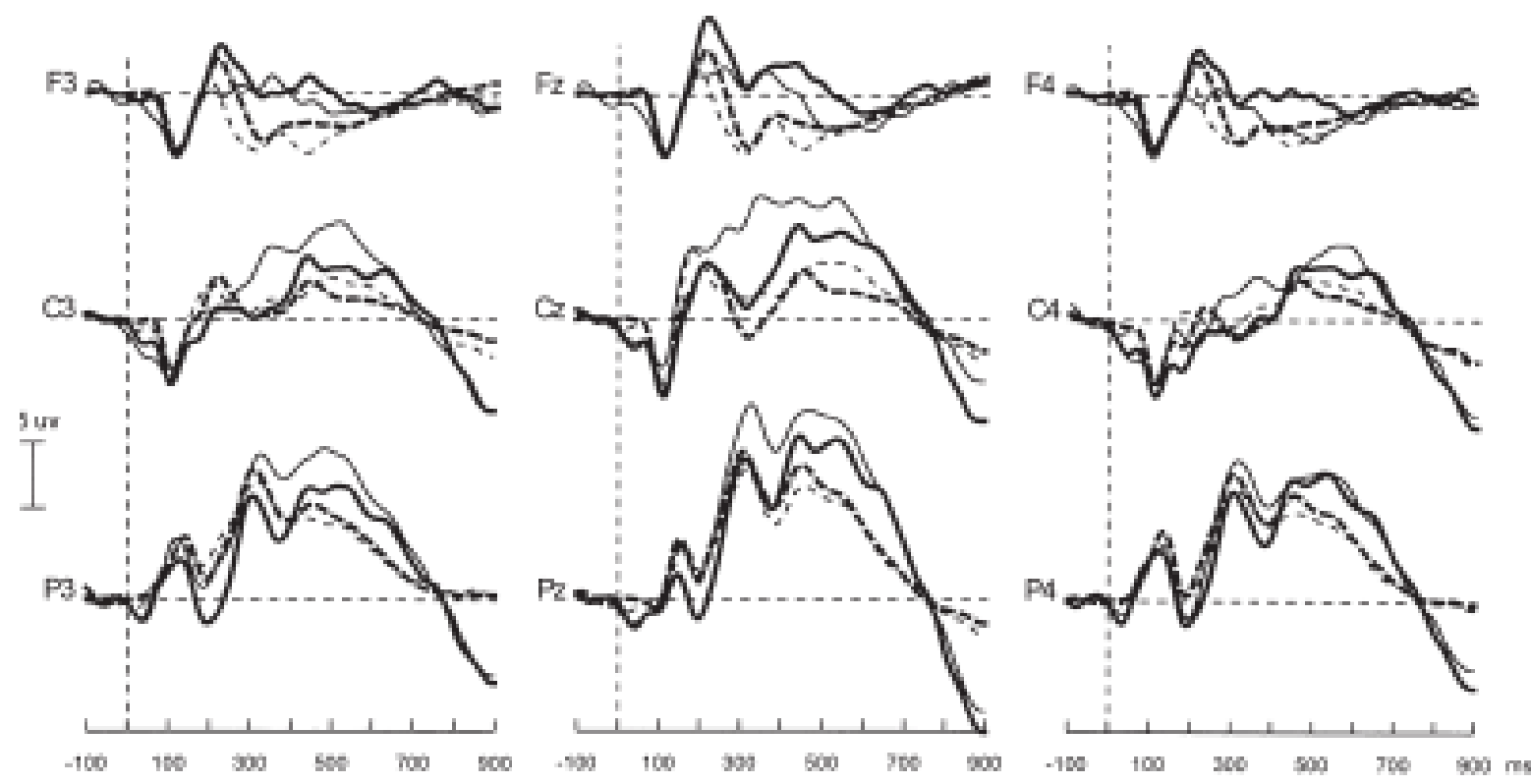

conting NONHATOHNG

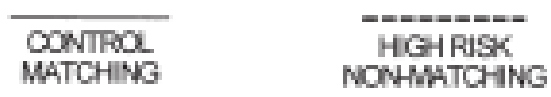

HICH RISK MATCHING

FIG. 1. Grand mean waveforms of the ERPs for the control $(n=19)$ and the HR $(n=17)$ groups. 


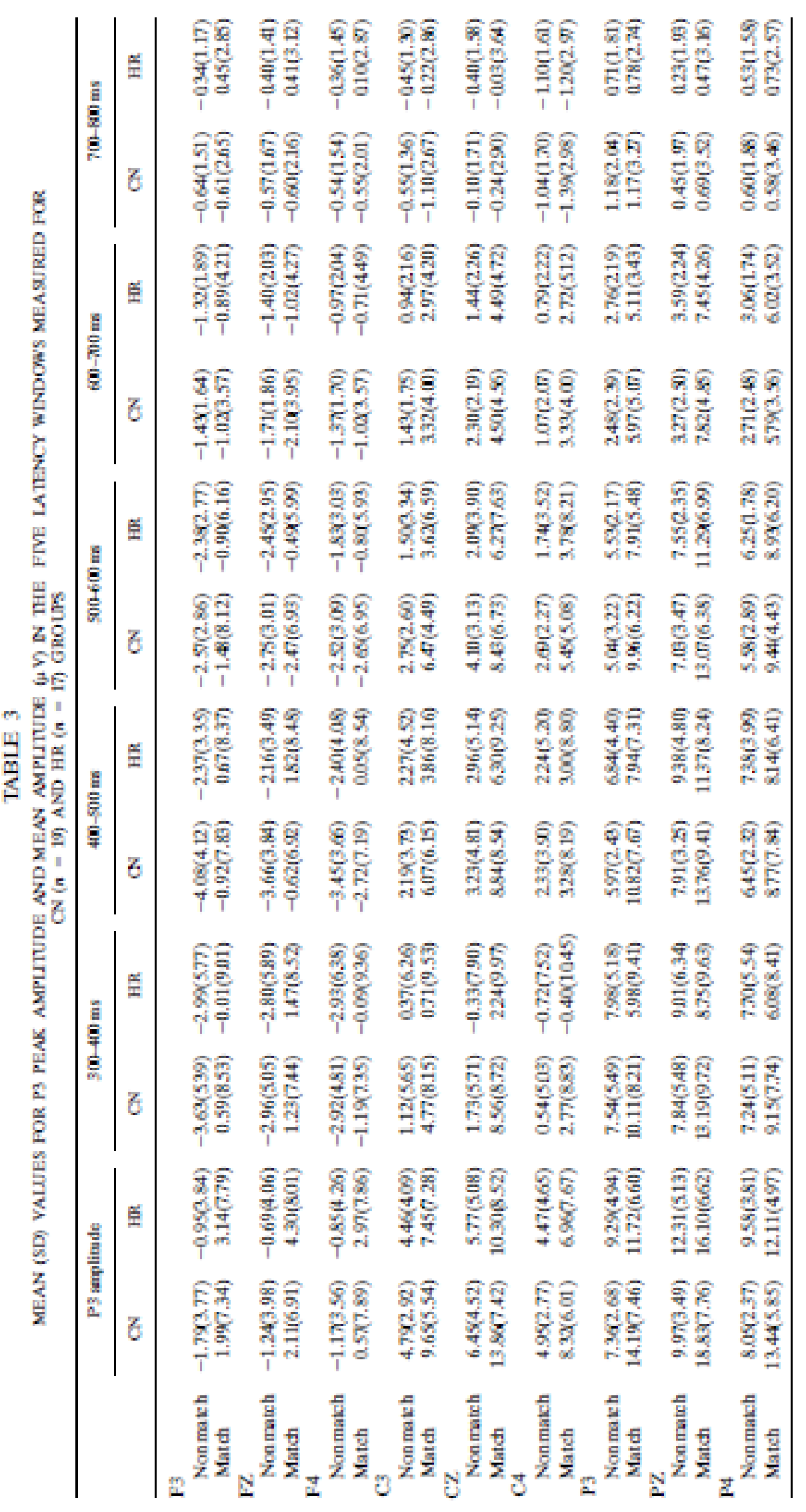

Post-print (final draft post-refereeing) 

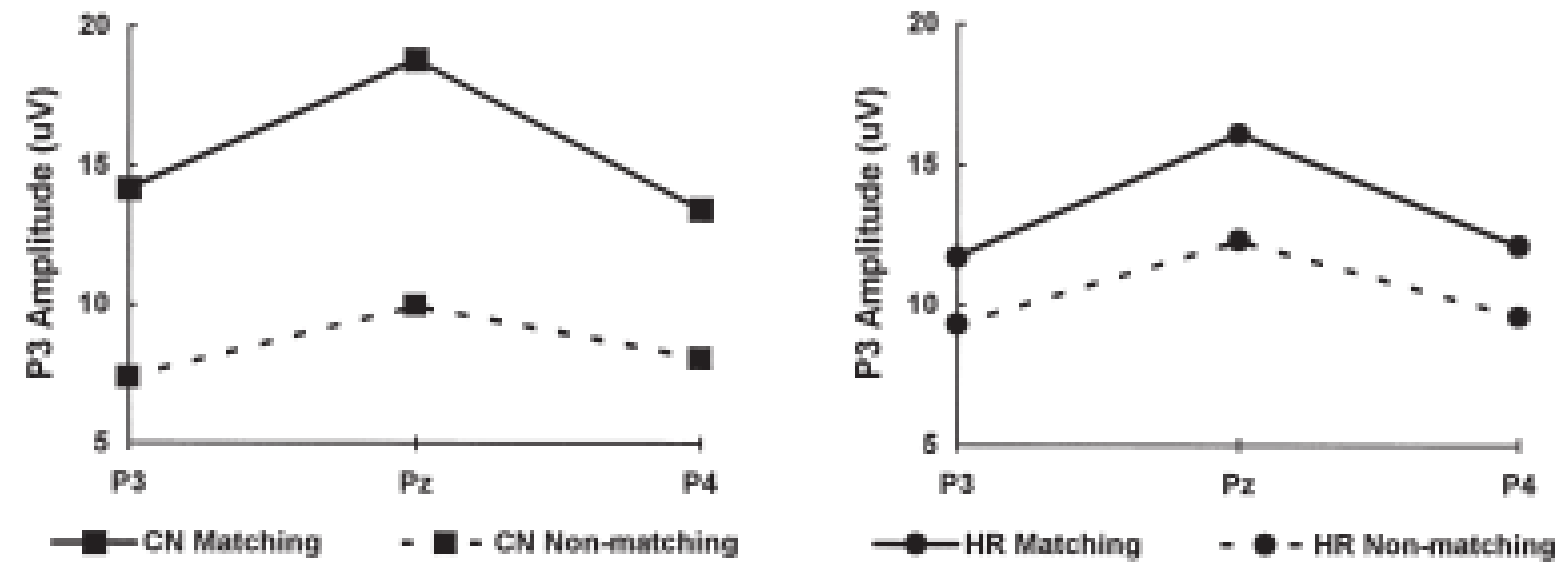

HIC. 2 Mean P3 amplitudes (PV) at the parietal regivn for the conirol (left) and the HR (right) groups. 